\title{
Predictors of In-Hospital Mortality in Isolated Total Anomalous Pulmonary Venous Connection
}

\author{
Eslam Elamry, Msc, ${ }^{1}$ Hesham Alkady, MD, ${ }^{2}$ Yasser Menaissy, $M D,{ }^{2}$ Osama Abdalla, MD $^{1}$ \\ ${ }^{1}$ Department of Cardiothoracic Surgery, Beni Suef University, Beni Suef, Egypt; ${ }^{2}$ Department of Cardiothoracic Surgery, Cairo \\ University, Egypt
}

\section{ABSTRACT}

Background: We assessed risk factors for early mortality in isolated total anomalous pulmonary venous connection over a modern era excluding emergent cases to eliminate the influence of associated factors on surgical outcome.

Methods: 70 patients with isolated total anomalous pulmonary venous connection who were repaired electively between January 2013 and February 2018 were included.

Results: In-hospital mortality was encountered in 4 patients $(5.7 \%)$. Upon univariate analysis, low age $(P=.003)$ and weight $(P=.001)$ at surgery, preoperative pulmonary venous obstruction $(P=.010)$, preoperative low oxygen saturation $(P=.031)$, long cardiopulmonary bypass $(P=.001)$ and aortic cross clamp $(P=.003)$ times, long duration of mechanical ventilation $(P=.001)$, chest infection $(P=0.041)$, postoperative low $\mathrm{CO}$ syndrome $(P<.001)$ and long postoperative inotropic support $(P=.015)$ were significant predictors of inhospital mortality. In multivariate analysis postoperative low cardiac output syndrome (OR: 1.060; 95\% CI: 1.008-1.116) and prolonged postoperative mechanical ventilation (OR: 1.772 ; 95\% CI: 1.141-2.751) were independent factors of inhospital mortality.

Conclusion: Surgical repair of TAPVC is now performed with acceptable results. According to our study, postoperative low cardiac output syndrome and prolonged postoperative mechanical ventilation were the most significant predictors for early mortality.

\section{INTRODUCTION}

Total anomalous pulmonary venous connection (TAPVC) is a rare heterogeneous group of congenital anomalies representing $1-3 \%$ of all congenital heart diseases with an incidence of about 1/100,000 live births [Herlong 2000]. Outcome of surgical repair of TAPVC is dependent on many variables such as early age at surgery, preoperative pulmonary venous obstruction (PVO), anatomic variations and associated anomalies with a rate of in-hospital mortality of about $5-10 \%$ [St Louis 2012]. In this study, we assess risk factors

Received Fanuary 29, 2019; March 12, 2019.

Correspondence: Hesham Alkady, Kasralaini Str, Almanial, Cairo, Egypt; +201006246611 (e-mail: heshamalqady@cu.edu.eg). for early mortality in isolated TAPVC over a modern era of 5 years, eliminating the influence of associated factors on the outcome of repair. Emergent cases were excluded due to the combined effects like higher incidence of preoperative PVO as well as more severity of symptoms in such cases.

\section{PATIENTS AND METHODS}

This study was approved by the ethical committee of our university. Data were collected from the hospital medical registers and outpatient records. An informed consent was obtained from at least one of the parents of the patients. 70 patients who underwent elective primary operative repair of isolated TAPVC between January 2013 and February 2018 at our institute were included in this study. Echocardiography and computed tomography angiography were performed on all patients to establish the diagnosis. Cases associated with cardiac anomalies other than patent ductus arteriosus (PDA) and atrial septal defect (ASD) as well as emergency cases were excluded. Emergency cases were defined as those requiring surgery within 24 hours of the established diagnosis. Early mortality was defined as death occurring during the same hospital stay after the primary operation. PVO was indicated by a non-phasic flow velocity $>1.8 \mathrm{~m} / \mathrm{s}$ or a pressure gradient along the pulmonary venous flow $>5 \mathrm{mmHg}$, either due to intrinsic stenosis, eg at the pulmonary venous ostia, or external stenosis at the connecting vein or atrial communication [Kato 2014]. Risks of early mortality were measured individually with patient parameters to assess their significance on the outcome of surgical repair. These factors included: age and weight at the time of initial surgery, gender, type of TAPVC, preoperative $\mathrm{PVO}$, preoperative $\mathrm{O} 2$ saturation, preoperative signs of heart failure, cardiopulmonary bypass (CPB) time, aortic cross-clamp (ACC) time, postoperative complications (pulmonary restenosis, arrhythmia, low cardiac output syndrome, chest infection, bleeding, and diaphragmatic paralysis), total hospital stay, duration of postoperative ventilation and duration of postoperative inotropic support. Low cardiac output syndrome was defined as persistent hypotension, oliguria or anuria, metabolic acidosis, and delayed peripheral capillary refill time with a cardiac index of less than $2.0 \mathrm{~L} / \mathrm{min} / \mathrm{m}^{2}$.

\section{Statistical Analysis}

Data were coded and entered into the statistical package SPSS (Statistical Package for the Social Sciences) version 
Table 1. Patient Characteristics $(n=70)$

\begin{tabular}{|c|c|}
\hline \multicolumn{2}{|l|}{ Sex, n (\%) } \\
\hline Male & $37(52.9)$ \\
\hline Female & $33(47.1)$ \\
\hline Median weight at surgery & $4.6 \mathrm{~kg}(3-10)$ \\
\hline Median age at surgery & $5 \mathrm{mo}(1.23-36)$ \\
\hline \multicolumn{2}{|l|}{ Type of TAPVC, n (\%) } \\
\hline Cardiac & $18(25.7)$ \\
\hline Supra-cardiac & $43(61.4)$ \\
\hline Infra-cardiac & $5(7.14)$ \\
\hline Mixed & $4(5.7)$ \\
\hline \multicolumn{2}{|l|}{ Pre-repair pulmonary venous obstruction, $\mathrm{n}(\%)$} \\
\hline Cardiac & $1(5.6)$ \\
\hline Supra-cardiac & $6(14)$ \\
\hline Infra-cardiac & $3(60)$ \\
\hline Mixed & $1(25)$ \\
\hline Preoperative signs heart failure, $\mathrm{n}(\%)$ & $42(60)$ \\
\hline Cyanosis, n (\%) & $28(40)$ \\
\hline Preoperative $\mathrm{O} 2$ saturation & $89.96 \pm 4.85$ \\
\hline \multicolumn{2}{|l|}{ Postoperative complications, n (\%) } \\
\hline Postoperative pulmonary restenosis & $3(4.3)$ \\
\hline Arrhythmia & $8(11.4)$ \\
\hline Postoperative low cardiac output syndrome & $3(4.3)$ \\
\hline Chest infection & $6(8.6)$ \\
\hline Bleeding & $3(4.3)$ \\
\hline Diaphragmatic paralysis & $3(4.3)$ \\
\hline Superficial wound infection & $2(2.8)$ \\
\hline
\end{tabular}

25. Data were summarized using mean \pm standard deviation, median, minimum and maximum (range) in quantitative data as well as frequency (count) and relative frequency (percentage) for categorical data. Univariate analysis and the significance of the association between quantitative variables were done using the non-parametric Mann-Whitney test. For comparing categorical data, Pearson chi-square test was performed and Fisher exact test was used instead when the expected frequency was less than 5. Multivariate logistic regression analysis was done to detect independent predictors of death. $P$ values less than .05 were considered statistically significant. Kaplan-Meier curves were used to determine the overall survival rate and freedom from pulmonary venous restenosis or reintervention.

\section{Surgical Techniques}

All operations were performed under general anesthesia through full median sternotomy. Cardiopulmonary bypass was established via aorto-bicaval cannulation. Myocardial protection was carried on with intermittent antegrade cold blood cardioplegia and systemic hypothermia to $28^{\circ} \mathrm{C}$. Deep

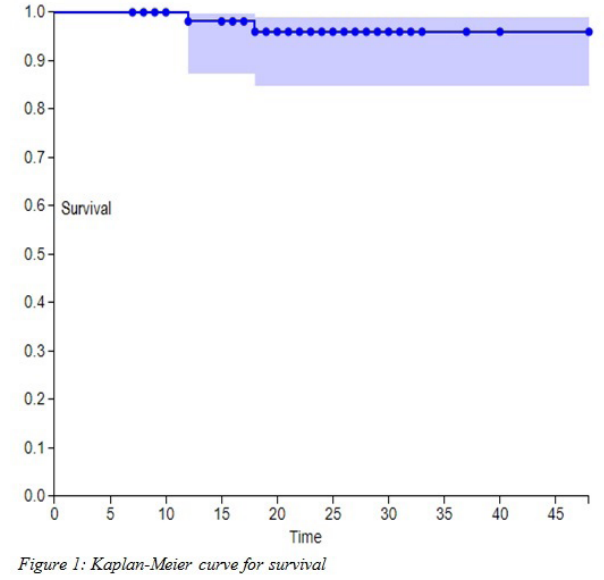

Figure 1. Kaplan-Meier curve for survival (time in months).

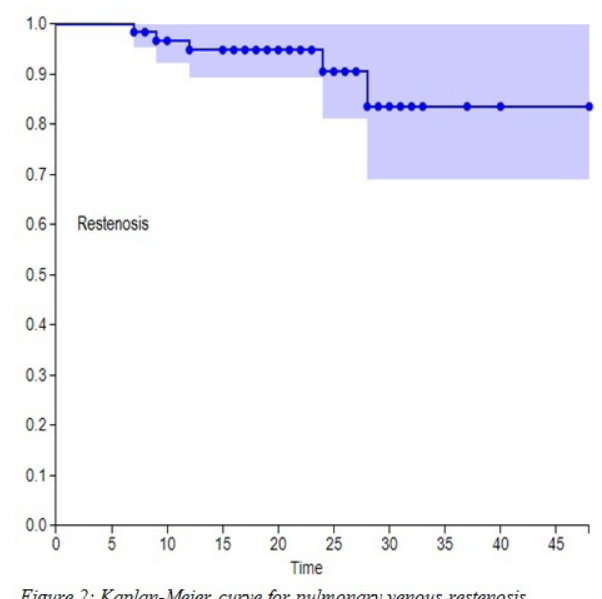

Figure 2. Kaplan-Meier curve for pulmonary venous restenosis (time in months).

hypothermic circulatory arrest was not used. The vertical vein was ligated in 68 patients (97\%). In only 2 cases the vein was left as a vent due to significantly high left atrial pressure. The median total cardiopulmonary bypass time for all patients was 90 minutes (range 48-180 minutes), median aortic cross clamp (ACC) duration was 60 minutes (range 30-90 minutes) and median total operative time was 130 minutes (range 95-240 minutes). A PDA was found in 32 patients (45.7\%) and was ligated.

Supra-cardiac type. The posterior wall of the left atrium (LA) was anastomosed to the pulmonary venous confluence side-to-side in a transverse fashion. The ASD was then closed through right atriotomy with a pericardial patch to enlarge the LA.

Cardiac type. The ASD was extended through right atriotomy to un-roof the coronary sinus, and was then closed using a pericardial patch to baffle the anomalous veins into the LA.

Infra-cardiac type. A vertical incision was made in the confluence of pulmonary veins and a matching incision was 
Table 2. Univariate Analysis of Patient Variables to Determine Significant Risks of Early Mortality

\begin{tabular}{|c|c|c|c|}
\hline & Yes & $(n=4)$ & No $(n=66)$ \\
\hline \multicolumn{4}{|l|}{ Sex, n (\%) } \\
\hline Male & $2(50)$ & $2(50)$ & \\
\hline Weight, $\mathrm{kg}$ & $3.67 \pm 0.48$ & $5.32 \pm 1.73$ & .002 \\
\hline \multicolumn{4}{|l|}{ TAPVC type, $\mathrm{n}(\%)$} \\
\hline Supra-cardiac & $2(50)$ & $40(60)$ & \\
\hline Cardiac & 0 & $17(26)$ & \\
\hline Infra-cardiac & $1(25)$ & $5(8)$ & \\
\hline Preoperative signs of heart failure, $n$ (\%) & $1(25)$ & $36(54.5)$ & .061 \\
\hline Cardiopulmonary bypass time, $\min$ & $128.57 \pm 37.05$ & $87.24 \pm 13.77$ & .007 \\
\hline Aortic cross-clamp time, min & $73.57 \pm 13.14$ & $57.60 \pm 10.36$ & .008 \\
\hline Postoperative pulmonary restenosis, n (\%) & $1(25)$ & $2(3)$ & .275 \\
\hline Arrhythmia, n (\%) & & $7(12.1)$ & .087 \\
\hline Postoperative low CO, n (\%) & $3(75)$ & 0 & .001 \\
\hline Chest infection, $\mathrm{n}(\%)$ & $1(25)$ & $5(7.5)$ & .041 \\
\hline Postoperative bleeding, n (\%) & 0 & $3(4.5)$ & .275 \\
\hline Diaphragmatic paralysis, n (\%) & 0 & $3(4.5)$ & .275 \\
\hline
\end{tabular}

CO indicates cardiac output; PVO, pulmonary venous obstruction. Bold indicates statistically significant values.

done on the posterior left atrial wall. Both incisions were then anastomosed side-to-side together. Finally, the ASD was closed using a pericardial patch.

Mixed type. A combination of the above techniques was used.

\section{RESULTS}

Thirty-seven cases were males $(52.9 \%)$ and 33 were females (47.1\%). Median age and weight at surgery were 5 months (range 1.23-36 months) and $4.6 \mathrm{~kg}$ (range 3-10 kg), respectively. The TAPVC anatomy was supra-cardiac in $43(61.4 \%)$ cases, cardiac in $18(25.7 \%)$, infra-cardiac in 5 $(7.14 \%)$, and mixed in $4(5.7 \%)$. In the supra-cardiac cases the vertical vein was found draining the venous confluence in the innominate vein in 25 patients, in the superior vena cava in 10 patients, and in the azygous vein in 8 patients; while in the cardiac form the venous confluence was connected to the coronary sinus in all cases. In the infra-cardiac variant, the vertical vein drained into the inferior vena cava in 2 patients and into the hepatic vein in 3 patients.

Eleven patients $(16 \%)$ were diagnosed with pre-repair pulmonary venous obstruction. Of these patients, 6 out of the 43 cases $(14 \%)$ with the supra-cardiac type of TAPVC, 1 out of the 18 cases $(5.6 \%)$ with the cardiac type, 3 out of the 5 cases $(60 \%)$ with the infra-cardiac type and 1 out of the 4 cases (25\%) with the mixed type were obstructed at the time of diagnosis. The 6 patients of supra-cardiac TAPVC with PVO had obstruction of the ascending vertical vein. The patient of cardiac TAPVC with PVO had obstruction at the atrial communication. In infra-cardiac TAPVC, one patient had obstruction of the vertical vein and the other 2 had obstruction in the intra-hepatic vein. The patient of mixed TAPVC with PVO had the obstruction at the vertical vein connecting the common pulmonary confluence to innominate vein. Forty-two patients $(60 \%)$ presented preoperatively, mainly 
Table 3. Most Significant Predictors of Early Mortality on Multivariate Analysis

\begin{tabular}{|c|c|c|c|c|c|c|c|c|}
\hline Postoperative ventilation & .572 & .224 & 6.496 & 1 & .011 & 1.772 & 1.141 & 2.751 \\
\hline Constant & 9.890 & 2.940 & 11.316 & 1 & .001 & 0.000 & & \\
\hline
\end{tabular}

$\mathrm{CO}$ indicates cardiac output.

with signs of heart failure. 28 patients $(40 \%)$ presented mainly with cyanosis. Preoperative O2 saturation (SO2) ranged between $76 \%$ and $95 \%$ with a mean of $89.96 \%$ (Table 1).

Some complications were encountered postoperatively (Table 1). The most frequent type of arrhythmia was nodal rhythm as it was reported in 5 patients, while sinus tachycardia was reported in 2 patients, and complete heart block occurred only in one case which was managed by permanent pacemaker. Four of the patients who developed arrhythmia were of supra-cardiac type $(4 / 43,9.3 \%), 3$ were of cardiac type (3/18, 16.7\%), and 1 was of mixed type (1/4, 25\%). PVO was found postoperatively in 3 patients (4.3\%). Two of these patients had stenosis at the anastomotic sites of the confluence to the left atrium and were managed with balloon dilatation without surgical intervention to be discharged home in a good general condition. The third patient had stenosis at 2 pulmonary veins before the confluence and was reoperated on during the same hospitalization with patch augmentation of the individual stenotic veins, but died later in the ICU. Wound infection was superficial (2 cases) and was managed conservatively with antibiotics according to culture and sensitivity and frequent dressings. Postoperative chest infection was a complication in 6 patients $(8.6 \%)$ and was significantly associated with increased duration of mechanical ventilation with $P<.001$. Patients with diaphragmatic paralysis (3 cases) received diaphragmatic placation on the side of paralysis through a postero-lateral thoracotomy.

Median postoperative ICU stay was 7 days (1-16 days). Median duration of mechanical ventilation in all patients was 1 day (range $0.5-13$ days) while that in patients who died was 8 days. On the other hand, median duration of postoperative inotropic support was 2 days (range 0.5-13 days) and in those who died was 8 days. Median postoperative total hospital stay was 8 days (1-20 days). In-hospital mortality reached 4 patients (5.7\%). The main cause of death was refractory low cardiac output (CO) syndrome in three cases and respiratory failure with inability to wean off mechanical ventilation due to chest infection followed by sepsis with multisystem organ dysfunction in one case.

Of the 66 patients who survived until discharge, 61 patients could be followed up with echocardiography for a period of 7 to 48 months (mean $26.87 \pm 10.46$ months). If echocardiographic findings were inconvenient, especially regarding the possibility of post-repair PVO, computed tomography angiography was performed. There were 2 late deaths at 12 and 18 months due to non-cardiac cause (gastroenteritis).
Five patients of the 61 followed cases (8.6\%) developed pulmonary venous stenosis. Two of these patients developed early postoperatively restenosis and were managed with balloon dilatation. These 2 patients were managed 24 and 28 months after the initial surgery, respectively, with patch augmentation and pulmonary vein endarterectomy due to worsening of symptoms (recurrent hemoptysis and decreasing exercise tolerance) and did well during the later follow up. The other 3 patients were not symptomatic and were managed with repeated balloon dilatation after a median period of 11.4 months from the primary repair at 1 year intervals (Figures 1 and 2).

Upon univariate analysis to determine significant risks of early mortality, lower age and weight at time of surgery, preoperative $\mathrm{PVO}$, lower preoperative oxygen saturation, longer $\mathrm{CBP}$ and ACC times, longer duration of mechanical ventilation, chest infection, postoperative low CO syndrome, and longer postoperative inotropic support were all found to be associated with increased mortality (Table 2).

Significant variables in mortality were entered in multivariate analysis to determine the independent predictors of in-hospital mortality. These were: the occurrence of postoperative low cardiac output syndrome and prolonged postoperative mechanical ventilation duration (Table 3).

\section{DISCUSSION}

The natural course of TAPVC without surgical treatment is associated with progressive heart failure, which is linked with $>80 \%$ mortality in the first year of life without surgery. Therefore, surgical correction is recommended once the diagnosis has been confirmed. Thanks to improvements in the diagnosis modalities, surgical techniques, and postoperative care regimens, surgical mortality of TAPVC patients decreased from $80 \%$ in early series to $5-10 \%$ in recent reports [Michielon 2002].

This mortality is influenced by anatomical factors and clinical aspects, as well as surgical issues. Most previous studies either represented a small cohort of individual centers or included cases with associated anomalies over a long period, which imposes a dramatic impact on the outcome [St Louis 2018; Shi 2017]. Therefore, in our cohort we included only cases with simple TAPVC over a recent period, minimizing the influence of associated anomalies as well as surgical eras on 
the early mortality. In addition, we excluded emergency cases which are associated with the highest mortality in most series due to combined factors like increased incidence of preoperative PVO and worsened symptoms of cyanosis (lower preoperative $\mathrm{O} 2$ saturation), as well as signs of heart failure which represent individual risk factors for early mortality [Karamlou 2007; Kanter 2006; Wu 2016; Kelle 2010]. To our knowledge, our study is thus unique in highly isolating risk factors of early mortality in simple TAPVC in elective patients.

According to our study, significant factors for mortality were low age and weight at the time of surgery, preoperative $\mathrm{PVO}$, preoperative low $\mathrm{O} 2$ saturation, long $\mathrm{CPB}$ time, long aortic cross clamp time, long postoperative mechanical ventilation, chest infection, postoperative low CO syndrome and long postoperative inotropic support.

Younger age as well as lower body weight at initial repair were noted to be significant risks for mortality in some studies [Karamlou 2007; Kanter 2006; Karaci 2012]. On the other hand, other studies like the study of Chi-Lun Wu and associates showed younger age at repair not to be a risk factor for mortality [Wu 2016]. There is a trend in pediatric cardiac surgery in general and in TAPVC in particular towards early repair once the diagnosis has been confirmed. However, whether younger age and lower weight represent a risk factor for mortality remains contradictory and is more related to center conditions and health care systems.

Preoperative pulmonary vein obstruction was reported to occur in $20-60 \%$ of TAPVC patients and to be more common in infra-cardiac and mixed types [Kelle 2010]. This was also true in our study with an incidence of $16 \%$ in our cases. PVO is linked in most studies, as well as ours, to both increased in-hospital mortality rate, and may be due to the need for emergent surgery, as well as increased incidence of postoperative pulmonary venous restenosis [Wu 2016; Karaci 2012; Husain 2012].

Lower preoperative initial $\mathrm{SO} 2$ and the presence of signs of heart failure may reflect the poor general condition of the patient and may point also to preoperative pulmonary obstruction or restrictive shunting of blood across the atrial communication. In our study, both factors were associated with higher mortality but only low preoperative O2 saturation was statistically significant. This agrees partly with the study of Chi-Lun $\mathrm{Wu}$ and associates, where patients with preoperative low $\mathrm{O} 2$ saturation, especially those requiring preoperative mechanical ventilation or inotropic support, showed higher mortality [Wu 2016].

Longer durations of cardiopulmonary bypass and aortic cross clamp as predictors of mortality are controversial among different studies. Both were significant risks for mortality in our study as well as the studies done by Gaynor et al and Husain et al [Husain 2012; Gaynor 1999]. In contrast, these factors were not shown as risk factors in the studies of Karamlou T and Wu CL. When assuming that all operations are done by the same experienced surgeons, long $\mathrm{CPB}$ and aortic cross clamp times are usually attributed to more complex procedures due to unfavorable anatomy especially if other anomalies are associated [Karamlou 2007; Wu 2016].
Long postoperative mechanical ventilation is usually attributed to the presence of chest infection or residual PVO. These were associated with higher mortality rate in our study as well as others [Wu 2016].

Similarly, the occurrence of postoperative low COP and the need of long postoperative inotropic support are closely related to increased mortality rate [Hancock Friesen 2005]. The occurrence of postoperative low cardiac output syndrome together with prolonged postoperative mechanical ventilation were the most significant risks for mortality upon multivariate analysis in our study. This agrees with the study done by Karamlou et al regarding postoperative low cardiac output syndrome [Karamlou 2007].

We did not find a significant association between the type of TAPVC and mortality $(P=.069)$. Some series showed a significant correlation between surgical mortality and the infra-cardiac group [Husain 2012]. Nevertheless, the impact of TAPVC type on mortality remains controversial between different studies according to the study cohort [Kirshbom 2002]. Similarly, post-repair pulmonary vein restenosis was not associated with increased early mortality in our study, in contrast to other studies, [Lacour-Gayet 2006] due to the low number of patients who developed such complications, as they were managed successfully.

Finally, this study represents a homogenous cohort of isolated TAPVC assessing individually risk factors of early mortality. This would help surgeons to explain potential risks and outcomes in TAPVC to the parents before surgery. However, our study carries the limitation of the retrospective nature and the individual center, pointing to the need for further prospective multi-institutional studies.

\section{Conclusion}

Surgical repair of TAPVC is now performed with acceptable results. According to our study, younger age and lower weight at repair, preoperative pulmonary venous obstruction, preoperative low $\mathrm{O} 2$ saturation, longer duration of $\mathrm{CPB}$ and ACC, longer duration of postoperative mechanical ventilation and inotropic are significant risk factors for in-hospital mortality. Postoperative low cardiac output syndrome and prolonged postoperative mechanical ventilation are the most significant predictors for mortality upon multivariate analysis.

\section{REFERENCES}

Gaynor JW, Collins MH, Rychik J, Gaughan JP, Spray TL. 1999. Longterm outcome of infants with single ventricle and total anomalous pulmonary venous connection. J Thorac Cardiovasc Surg 117:506-13.

Hancock Friesen CL, Zurakowski D, Thiagarajan RR, et al. 2005. Total anomalous pulmonary venous connection: an analysis of current management strategies in a single institution. Ann Thorac Surg 79:596-606.

Herlong JR, Jaggers JJ, Ungerleider RM. 2000. Congenital heart surgery nomenclature and database project: pulmonary venousanomalies. Ann Thorac Surg 69:56-69.

Husain SA, Maldonado E, Rasch D, et al. 2012. Total anomalous pulmonary venous connection: factors associated with mortality and recurrent pulmonary venous obstruction. Ann Thorac Surg 94:825-32. 
Kanter KR. 2006. Surgical repair of total anomalous pulmonary venous connection. Semin Thorac Cardiovasc Surg Pediatr Card Surg Annu 9:40-4.

Karaci AR, Harmandar B, Aydemir NA, Sasmazel A, Balci AY. 2012. Early and intermediate term results for surgical correction of total anomalous pulmonary venous connection. J Card Surg 27: 376-80.

Karamlou T, Gurofsky R, Al Sukhni E, et al. 2007. Factors associated with mortality and reoperation in 377 children with total anomalous pulmonary venous connection. Circulation 115: 1591 .

Kato H, Fu YY, Zhu J, Wang L, et al. 2014. Pulmonary vein stenosis and the pathophysiology of "upstream" pulmonary veins. J Thorac Cardiovasc Surg 148:245-53.

Kelle AM, Backer CL, Gossett JG, Kaushal S, Mavroudis C. 2010. Total anomalous pulmonary venous connection: results of surgical repair of 100 patients at a single institution. J Thorac Cardiovasc Surg 139:1387.

Kirshbom PM, Myung RJ, Gaynor JW, et al. 2002. Preoperative pulmonary venous obstruction affects long-term outcome for survivors of total anomalous pulmonary venous connection repair. Ann Thorac Surg 74:1616-20.
Lacour-Gayet F. 2006. Surgery for pulmonary venous obstruction after repair of total anomalous pulmonary venous return. Semin Thorac Cardiovasc Surg Pediatr Card Surg Annu 45-50.

Michielon G, Di Donato RM, Pasquini L, et al. 2002. Total anomalous pulmonary venous connection: long-term appraisal with evolving technical solutions. Eur J Cardiothorac Surg 22: 184-91.

Shi G, Zhu Z, Chen J, et al. 2017. Total anomalous pulmonary venous connection: the current management strategies in a pediatric cohort of 768 patients. Circulation 135:48-58.

St Louis JD, Harvey BA, Menk JS, et al. 2012. Repair of "simple" total anomalous pulmonary venous connection: a review from the Pediatric Cardiac Care Consortium. Ann Thorac Surg 94:133-7.

St Louis JD, McCracken CE, Turk EM, et al. 2018. Long-term transplant-free survival after repair of total anomalous pulmonary venous connection. Ann Thorac Surg 105:186-92.

Wu CL, Kan CD, Wu JM, Wang JM. 2016. Risk factors of total anomalous pulmonary venous connection surgery. Ped Health Res 1:1-7. 